\title{
Determining factors for carotid mean/max intima-media thickness and brachial flow-mediated dilation in healthy young women
}

\author{
Shiro Hoshida, Takashi Miki, Takafumi Nakagawa, Yukinori Shinoda, Takayoshi Adachi \\ Division of Cardiovascular Medicine, Yao Municipal Hospital, Yao, Japan \\ Email: hoshidas@city.yao.osaka.jp
}

Received 13 February 2012; revised 14 March 2012; accepted 24 March 2012

\begin{abstract}
Background: Many factors can contribute to atherosclerotic-type vascular changes in older individuals or men. Thus, confining the investigation to young women with no clinical evidence of the condition could enhance understanding of the early stages of cardiovascular disease. The aim of this study was to determine whether carotid mean/max intima-media thickness (IMT) and brachial flow-mediated dilation (FMD) values, which are well-known event-related indices, are associated with laboratory data and the other vascular indices of atherosclerosis in healthy young women. Methods: Carotid mean/max IMT and brachial FMD were measured in young women with no clinical evidence of atherosclerosis $(n=110$; mean age, 39 years) who were instructed not to eat, drink or smoke after 9 PM the evening before testing. All participants also underwent laboratory assessment, including simultaneous measurements of arterial stiffness such as augmentation index (AI), cardio-ankle vascular index (CAVI) and brachial-ankle pulse wave velocity (baPWV). Results: Mean IMT was significantly and positively associated with age $(p=0.002)$, CAVI $(p=0.044)$, low-density lipoprotein-cholesterol (LDL-C, $p=0.047$ ) and high-sensitive C-reactive protein (hs-CRP, $p=0.002$ ) values but was not related to FMD, AI, baPWV or triglycerides (TG) in the multivariate regression analysis. Similarly, max IMT was positively associated with age $(p<0.001)$, LDL-C ( $p=$ $0.003)$ and hs-CRP $(p=0.005)$ values but was not related to FMD, AI, CAVI, baPWV, TG or blood pressure level in the multivariate regression analysis. The association between LDL-C and max IMT was much stronger than that between LDL-C and mean IMT. Brachial FMD was positively associated only with heart rate in the multivariate regression analysis. Conclusions: These results suggest that mean IMT
\end{abstract}

*The authors declare that there is no conflict of interest. more closely represents the sclerotic aspect of vascular change, whereas max IMT represents the atherotic aspect in healthy young women. Although the relationship between the autonomic nervous system and heart rate is well-known, there may be a complex interaction between the autonomic nervous system and endothelial function.

Keywords: Intima-Media Thickness; Flow-Mediated Dilation; Young Women

\section{INTRODUCTION}

The incidence of systemic atherosclerotic disease has increased as the elderly population has increased. Many noninvasive devices can be used to evaluate serial changes in the extent of atherosclerosis, allowing early detection of the disease and a reduction in cardiovascular events. The intima-media thickness (IMT) of the carotid artery, which is determined noninvasively with ultrasonography, is an early predictor of general arteriosclerosis and is useful for gauging the extent of subclinical atherosclerosis [1,2]. Serum cholesterol level [3] and hypertension [4] have been inversely associated with endothelial function, as assessed by brachial artery flow-mediated dilation (FMD), in prior studies. Increased arterial stiffness has also been associated with heart attacks and strokes. Pulse wave velocity (PWV), which is measured using automatic devices, is widely accepted as an indicator of arterial stiffness and a risk marker for cardiovascular disease $[5,6]$. When compared with the augmentation index (AI), another common measure of arterial stiffness, PWV was more closely associated with cardiovascular disease in relatively low-risk populations [7]. The cardio-ankle vascular index (CAVI) was recently introduced as another indicator of arterial stiffness. Specifically, CAVI measures the stiffness parameter beta of the thoracic descending aorta [8], which is independent of blood pressure. CAVI is a marker of atherosclerosis and could quantita- 
tively reflect the condition of the large arteries [9].

Many factors can contribute to atherosclerotic-type vascular changes in older individuals or men. Thus, confining the investigation to young women with no clinical evidence of the condition could enhance understanding of the early stages of cardiovascular disease. The aim of this study was to determine whether carotid mean/max IMT and brachial FMD values, which are well-known cardiovascular event-related indices $[10,11]$, are associated with laboratory data and the other vascular indices for atherosclerosis, such as AI, CAVI and PWV, in healthy young women.

\section{METHODS}

Women ( $n=110$; mean age, 39 ) who participated in the study were instructed not to eat, drink or smoke after 9 PM the evening before testing. Participants were examined in the early morning (between 8:00 and 9:00 AM) in the fasted state after resting quietly for 15 minutes. Standard laboratory biochemical measurements were performed on blood collected from the participants, all of whom were subjects at our hospital. None of the participants had received treatment for hypertension, hyperlipidemia or diabetes mellitus. Participants who had smoked in the last 30 days were classified as smokers, whereas previous smokers and patients who have never smoked were considered nonsmokers in this study.

For each patient, the IMT and stiffness parameter beta of the carotid artery were measured using an ultrasound SSD- $\alpha 10$ machine (ALOKA Co. Ltd., Tokyo, Japan) with a 7.5-MHz transducer. The extracranial common carotid artery, the carotid bulb, and the internal carotid artery in the neck were scanned bilaterally from longitudinal and transverse projections. IMT, which was defined as the distance between the lumen-intima interface and the medial-adventitial interface, was measured at the thickest point [i.e., maximum IMT (max IMT)] and at two adjacent points (located $1 \mathrm{~cm}$ upstream and $1 \mathrm{~cm}$ downstream from this site). These measurements were subsequently averaged to obtain the mean IMT. The average of the right and left values of max IMT and mean IMT was used as the individual value [12]. No localized elevated lesions with a maximum thickness of more than $1 \mathrm{~mm}$ (i.e., plaques) were found on the surface of the intimamedia complex. The stiffness parameter beta of the carotid artery, which indicates blood pressure-independent, patient-specific vascular stiffness, was measured at a point where the max IMT was not evaluated.

After measurements of mean IMT, max IMT and stiffness parameter beta were obtained, endothelium-dependent FMD of the brachial artery was measured using an established noninvasive method based on recent guidelines of the European Society of Hypertension (2007)
[13], which were slightly modified for the purposes of this study. While each participant was in the supine position, her right arm was used to monitor blood pressure level and pulse rate at 5-minute intervals throughout the examination. A standard pediatric cuff was positioned around the left arm $5 \mathrm{~cm}$ below the antecubital fossa. An SSD- $\alpha 10$ machine was used to acquire ultrasonographic images of the left brachial artery. After baseline images were obtained for 2 minutes, the pediatric cuff was inflated to $50 \mathrm{~mm} \mathrm{Hg}$ above the participant's systolic blood pressure level to occlude the left brachial artery. The pediatric cuff was kept inflated for 5 minutes. Images of the left brachial artery were then captured continuously for 3 minutes after cuff deflation, and diameter measurements were captured in diastole. Brachial FMD was calculated as reported previously [11].

The brachial-ankle PWV (baPWV), CAVI and AI were examined as measures of arterial stiffness. Blood pressure and baPWV values were studied using a BP203RPE II unit (OMRON HEALTHCARE Co. Ltd., Kyoto, Japan). The CAVI was measured noninvasively using the VS-1500 system (FUKUDA DENSHI Co. Ltd., Tokyo, Japan), and the stiffness parameter beta was then entered into an equation for determining vascular elasticity and PWV [9]. The AI, which was measured using a HEM-9000AI (OMRON HEALTHCARE Co. Ltd., Kyoto, Japan), was defined as the ratio of augmentation to central blood pressure and was expressed as a percentage.

\section{STATISTICAL ANALYSIS}

Univariate analysis was first performed using a Pearson correlation coefficient and regression analysis to evaluate related factors for mean IMT, max IMT and FMD. Statistical significance was subsequently assessed with multivariate regression analysis using significantly related factors from the univariate analysis as the covariates to elucidate independent determinants of mean IMT, max IMT and FMD. All $p$ values were two-tailed. The correlation between brachial artery diameter and FMD or heart rate was evaluated using a Pearson product moment correlation coefficient, and the $p$ value was examined with regression analysis.

\section{RESULTS}

The mean \pm standard deviation values are shown in Table 1; all values were within the normal range.

\subsection{Univariate Analysis}

Univariate analysis identified potential associations with mean IMT and max IMT or FMD. Mean IMT was positively associated with age, AI, CAVI, baPWV and serum 
Table 1. Characteristics of this study's subjects.

\begin{tabular}{lcc}
\hline Age & $39 \pm 9$ & years \\
Smoking -/+ & $78 / 32$ & \\
Systolic blood pressure & $110 \pm 12$ & $\mathrm{~mm} \mathrm{Hg}$ \\
Diastolic blood pressure & $72 \pm 10$ & $\mathrm{~mm} \mathrm{Hg}$ \\
Heart rate & $65 \pm 8$ & beats/min \\
Mean IMT & $0.52 \pm 0.10$ & $\mathrm{~mm}$ \\
Max IMT & $0.58 \pm 0.13$ & $\mathrm{~mm}$ \\
FMD & $7.5 \pm 2.5$ & $\%$ \\
Stiffness parameter beta & $6.1 \pm 1.8$ & \\
Augmentation index & $77 \pm 14$ & $\%$ \\
CAVI & $6.7 \pm 1.0$ & \\
baPWV & $1155 \pm 173$ & $\mathrm{~cm} / \mathrm{sec}$ \\
LDL-C & $116 \pm 28$ & $\mathrm{mg} / \mathrm{dl}$ \\
HDL-C & $70 \pm 14$ & $\mathrm{mg} / \mathrm{dl}$ \\
Triglycerides & $76 \pm 32$ & $\mathrm{mg} / \mathrm{dl}$ \\
hs-CRP & $0.03 \pm 0.04$ & $\mathrm{mg} / \mathrm{dl}$ \\
\hline
\end{tabular}

Data are mean \pm SD or the number of subjects.

levels of low-density lipoprotein-cholesterol (LDL-C), triglycerides (TG) and high-sensitive C-reactive protein (hs-CRP) and was inversely associated with FMD (Table 2(a)). Similar associations were found for max IMT in addition to a positive association with systolic and diastolic blood pressure levels (Table 2(b)). In contrast, brachial FMD was positively associated only with heart rate and was inversely associated with age, as well as the mean IMT (Table 2(c)). When we substituted the max IMT data in place of the mean IMT data, FMD was similarly inversely associated with this factor (data not shown). Baseline brachial artery diameter was inversely correlated with FMD, although it did not correlate with heart rate (Table 3).

\subsection{Multivariate Analysis}

In each multivariate analysis, significantly related factors from the univariate analysis were used as the covariates. Among those factors, mean IMT was positively associated with age ( $p=0.002)$, CAVI $(p=0.044)$, LDL-C ( $p$ $=0.047)$ and hs-CRP $(p=0.002)$ but was not related to FMD, AI, baPWV or TG in the multivariate regression analysis (Table 2(a)). Similarly, among significantly related factors from the univariate analysis, max IMT was positively associated with age $(p<0.001)$, LDL-C ( $p$ $=0.003)$ and hs-CRP $(p=0.005)$ but was not related to FMD, AI, CAVI, baPWV, TG or blood pressure in the multivariate regression analysis (Table 2(b)). The association between LDL-C and max IMT was much stronger than the association between LCL-C and mean IMT.

Brachial FMD was positively associated only with heart rate in the multivariate regression analysis $(p=$
0.017) (Table 2(c)). FMD was persistently associated only with heart rate when we substituted the max IMT data in place of the mean IMT data $(p=0.020)$ or the data from both IMTs $(p=0.015)$.

\section{DISCUSSION}

In this study, we verified the association between several common ultrasonographic parameters and other indices of vascular atherosclerosis and laboratory data in healthy young women with no clinical evidence of atherosclerosis. In multivariate modeling, increasing age and increasing levels of LDL-C and hs-CRP were associated with increased mean IMT and max IMT, although the association between LDL-C and max IMT was much stronger than that between LDL-C and mean IMT. Furthermore, as a measure of arterial stiffness, CAVI was persistently associated with increased mean IMT but not with increased max IMT. FMD was associated with increased heart rate.

\subsection{Carotid IMT}

Confining our investigation to subjects with no clinical evidence of atherosclerosis allowed examination of atherosclerosis early in the natural history of cardiovascular disease. Multiple regression analysis revealed that age, LDL-C and hs-CRP levels were positive factors for both mean IMT and max IMT in young women. Age was the most important determinant identified, although our evidence is insufficient to determine whether age relates to cumulative exposure to cardiovascular risk factors. At the very least, low-grade inflammation strongly contributes to early stage carotid atherosclerosis assessed with mean IMT or max IMT in young subjects. Although LDL-C level was significantly associated with both mean IMT and max IMT, the extent of the association was clearly different: LDL-C levels were closely associated with max IMT but only mildly associated with mean IMT. In previous studies with healthy subjects, age [14], dyslipidemia [14], hs-CRP [15], systolic blood pressure level [16] and smoking history [16] were shown to be important independent predictors of IMT. However, the difference in the determining factors between the mean IMT and the max IMT remains to be seen in these studies. Moreover, these studies did not include other indices for the noninvasive assessment of vascular atherosclerosis. In our study, CAVI was significantly associated with mean IMT but not with max IMT. These findings described above suggest that mean IMT more closely represents the sclerotic aspect of vascular change, whereas max IMT represents the atherotic aspect in our subjects. One could reveal these differences because our subjects are young women and smoking may be less effective on atherosclerotic change under these conditions. In the case 
Table 2. Univariate and multivariate analyses. Univariate analysis was first performed using a Pearson correlation coefficient and regression analysis to evaluate related factors for mean IMT (a), max IMT (b) and FMD (c). Statistical significance was subsequently assessed with multivariate regression analysis using significantly related factors from the univariate analysis as the covariates to elucidate independent determinants of mean IMT, max IMT and FMD.

(a)

\begin{tabular}{lccccc}
\hline & \multicolumn{2}{c}{ Univariate } & \multicolumn{3}{c}{ Multivariate } \\
\hline & $r$ value & $p$ value & $p$ value & $\beta$ & $95 \%$ CI \\
Age & 0.465 & $<0.001$ & 0.002 & 0.005 & $(0.002-0.008)$ \\
Smoking & -0.015 & 0.879 & - & - & - \\
Systolic blood pressure & 0.151 & 0.215 & - & - & - \\
Diastolic blood pressure & 0.164 & 0.113 & - & - & - \\
Heart rate & -0.083 & 0.284 & - & - & - \\
FMD & -0.236 & 0.044 & 0.309 & -0.003 & $(-0.010-0.003)$ \\
Stiffness parameter beta & 0.089 & 0.698 & 0.285 & - \\
Augmentation index & 0.409 & 0.003 & 0.044 & $<-0.001$ & $(-0.003-<0.001)$ \\
CAVI & 0.485 & $<0.001$ & 0.646 & $<-027$ & $(<0.001-0.053)$ \\
baPWV & 0.403 & 0.002 & 0.047 & $<0.001$ & $(<-0.001-0.001)$ \\
LDL-C & 0.424 & $<0.001$ & - & - & $(<0.001-0.001)$ \\
HDL-C & -0.134 & 0.248 & 0.067 & - \\
Triglycerides & 0.345 & $<0.001$ & 0.002 & $<0.001$ & $(<-0.001-0.001)$ \\
hs-CRP & 0.253 & 0.007 & & 0.647 & $(0.249-1.045)$ \\
\hline
\end{tabular}

$\beta$; standardized regression coefficient. CI; confidential interval.

(b)

\begin{tabular}{lccccc}
\hline & \multicolumn{2}{c}{ Univariate } & & \multicolumn{2}{c}{ Multivariate } \\
\hline & $r$ value & $p$ value & $p$ value & $\beta$ & $95 \%$ CI \\
Age & 0.573 & $<0.001$ & $<0.001$ & 0.007 & $(0.004-0.011)$ \\
Smoking & 0.069 & 0.474 & - & - & - \\
Systolic blood pressure & 0.216 & 0.023 & 0.182 & 0.002 & $(-0.001-0.006)$ \\
Diastolic blood pressure & 0.276 & 0.003 & 0.682 & -0.001 & $(-0.006-0.004)$ \\
Heart rate & -0.089 & 0.350 & - & - & - \\
FMD & -0.208 & 0.029 & 0.572 & -0.002 & $(-0.010-0.006)$ \\
Stiffness parameter beta & 0.056 & 0.352 & - & - \\
Augmentation index & 0.269 & $<0.001$ & 0.713 & $<-0.001$ & $(-0.003-0.002)$ \\
CAVI & 0.406 & $<0.001$ & 0.139 & 0.024 & $(-0.008-0.056)$ \\
baPWV & 0.295 & $<0.001$ & 0.933 & $<0.001$ & $(<-0.001-<0.001)$ \\
LDL-C & 0.349 & $<0.001$ & 0.003 & 0.001 & $(<0.001-0.002)$ \\
HDL-C & -0.118 & 0.164 & - & - & - \\
Triglycerides & 0.366 & $<0.001$ & 0.522 & $<0.001$ & $(<-0.001-<0.001)$ \\
hs-CRP & 0.275 & 0.008 & 0.005 & 0.709 & $(0.218-1.199)$ \\
\hline
\end{tabular}

(c)

\begin{tabular}{lccccc}
\hline & \multicolumn{2}{c}{ Univariate } & & \multicolumn{2}{c}{ Multivariate } \\
\hline & $r$ value & $p$ value & $r$ value & $p$ value & $r$ value \\
Age & -0.224 & 0.018 & 0.244 & -0.059 & $-0.160-0.041)$ \\
Smoking & 0.017 & 0.860 & - & - & - \\
Systolic blood pressure & 0.019 & 0.841 & - & - & - \\
Diastolic blood pressure & $<-0.001$ & 0.997 & - & - & $(0.018-0.181)$ \\
Heart rate & 0.232 & 0.015 & 0.017 & 0.099 & $(-9.816-3.055)$ \\
Mean IMT & -0.236 & 0.044 & -9.309 & - \\
Stiffness parameter beta & 0.019 & 0.361 & - & - & - \\
Augmentation index & -0.014 & 0.888 & - & - & - \\
CAVI & -0.145 & 0.129 & - & - & - \\
baPWV & 0.012 & 0.897 & - & - & - \\
LDL-C & -0.177 & 0.064 & - & - & - \\
HDL-C & 0.021 & 0.831 & - & - & - \\
Triglycerides & -0.171 & 0.074 & - & - \\
hs-CRP & -0.006 & 0.947 & & - & - \\
\hline
\end{tabular}


Table 3. Involvement of diameter of brachial artery and width of flow-mediated dilation for brachial flow-mediated dilation (a) and heart rate (b) by a correlation coefficient ( $r$ value) and regression analysis ( $p$ value).

(a)

\begin{tabular}{lcc}
\hline & $r$ value & $p$ value \\
\hline Diameter of brachial artery $(\mathrm{mm})$ & -0.509 & $<0.001$ \\
Width of flow-mediated dilation $(\mathrm{mm})$ & 0.842 & $<0.001$ \\
\hline
\end{tabular}

(b)

\begin{tabular}{lcc}
\hline & $r$ value & $p$ value \\
\hline Diameter of brachial artery $(\mathrm{mm})$ & -0.041 & 0.671 \\
Width of flow-mediated dilation $(\mathrm{mm})$ & 0.119 & 0.213 \\
\hline
\end{tabular}

of older individuals or males, many factors may contribute to both sclerotic and atherotic aspects of vascular change, making the distinction less clear.

Although AI, stiffness parameter beta of the carotid artery and baPWV were not associated with either IMT index, the CAVI was significantly associated with mean IMT. This result indicates that CAVI may be superior to the other indices in evaluating the extent of arterial stiffness in the early stages of cardiovascular disease.

\subsection{Brachial FMD}

Endothelial dysfunction is an important early event in atherogenesis [17,18], and brachial FMD is a well-known marker of endothelial function. In our subjects with subclinical atherosclerosis, FMD was positively associated only with heart rate. No measure of adiposity predicted FMD. This result may be attributable to the relatively low LDL-C levels of our subjects; fewer than $20 \%$ of our subjects had an LDL-C level $>140 \mathrm{mg} / \mathrm{dl}$. In healthy subjects, triglyceride level has been a significant determinant of endothelial function [16], but this study did not evaluate heart rate. In the Framingham Heart Study, the significant multivariate correlates of brachial FMD were age, gender, systolic blood pressure and heart rate [19], although mean age (61 years) and systolic blood pressure level $((122 \pm 18) \mathrm{mm} \mathrm{Hg})$ of the participants were much higher and mean brachial FMD (3.3\%) was much lower than those of our subjects, who were all young women. Importantly, mean heart rate for both studies was $65 \mathrm{bpm}$, thereby reinforcing our finding that FMD was positively associated only with heart rate.

In general, the association between resting heart rate and cardiovascular disease has been documented in a number of epidemiologic and clinical studies [20]. In the other study with younger participants (mean age, 36 years), brachial FMD was inversely associated with older age, male gender and larger vascular size, but it was not associated with heart rate [21]. Medications that lower heart rate have showed variable effects on FMD [22,23]. Although the association between heart rate and brachial FMD observed in our study and in the Framingham Heart Study was not observed in the research efforts described above, the difference may relate to the characteristics of the populations that were studied (i.e., the underlying level of subclinical or clinical atherosclerosis may have been different). The determining factors for brachial FMD may vary between normal and abnormal ranges of FMD.

Basal brachial artery diameter reportedly is inversely associated with FMD [19], which is a finding that was also confirmed by our study. However, the correlation between basal brachial artery diameter and heart rate was not significant. This result indicates that the association between FMD and heart rate is not related to brachial artery diameter. In subjects whose vasculature releases a higher level of nitric oxide, potentially producing a higher FMD, peripheral vasculature may be dilated more extensively, leading to increased heart rate. Furthermore, higher-frequency pulsatile flow is reportedly associated with increased release of nitric oxide in animal models [24], which is possibly related to the positive association between FMD and heart rate in our study. Although the relationship between the autonomic nervous system and heart rate is well-known, the interaction between the autonomic nervous system and vascular tone/endothelial function is complex [25].

\subsection{Limitations}

The results of our study cannot be extrapolated to the general population because we enrolled only young women with no history of medication to treat hypertension, diabetes or dyslipidemia. Gender-related confounding factors may also be involved. Because we measured baPWV instead of carotid-femoral PWV, the strength of CAVI versus carotid-femoral PWV as a factor in mean IMT was not examined. We also have no data regarding vascular response to nitroglycerin. Therefore, we cannot state unequivocally whether the observed variations in brachial FMD were due to endothelial dysfunction or to an impaired vascular smooth muscle cell response. However, studies in younger subjects have found no evidence of impairment in response to nitroglycerin [26]. Although variations in endothelial function during the menstrual cycle have been reported [27], we did not regulate the cycle for FMD examination.

\subsection{Clinical Implications}

This study is the first to show that the determining factors between mean IMT and max IMT are different and heart rate is the only determining factor for FMD in healthy young women with subclinical atherosclerosis. 
However, our study has a pilot nature: the sample size was so small for a study of this kind. The preliminary data obtained from our study probably will guide the clinicians to perform large-scale prospective clinical studies.

\section{CONCLUSION}

Our findings suggest that mean IMT more closely represents the sclerotic aspect of vascular change, whereas max IMT represents the atherotic aspect in healthy young women. Although the relationship between the autonomic nervous system and heart rate is well-known, there may be a complex interaction between the autonomic nervous system and endothelial function.

\section{REFERENCES}

[1] Pignoli, P., Tremoli, E., Poli, A., et al. (1986) Intimal plus medial thickness of the arterial wall: A direct measurement with ultrasound imaging. Circulation, 74, 13991406. doi:10.1161/01.CIR.74.6.1399

[2] Stein, J.H., Korcarz, C.E., Hurst, R.T., et al. (2008) Use of carotid ultrasound to identify subclinical vascular disease and evaluate cardiovascular disease risk: A consensus statement from the American society of echocardiogramphy carotid intima-media thickness task force. Endorsed by the society for vascular medicine. Journal of American Society of Echocardiography, 21, 93-111.

[3] Clarkson, P., Celermajer, D.S., Donald, A.E., et al. (1996) Impaired vascular reactivity in insulin-dependent diabetes mellitus is related to disease duration and low density lipoprotein cholesterol levels. Journal of American College of Cardiology, 28, 573-579. doi:10.1016/0735-1097(96)82380-1

[4] Gokce, N., Holbrook, M., Duffy, S.J., et al. (2001) Effects of race and hypertension on flow-mediated and nitroglycerin-mediated dilation of the brachial artery. $\mathrm{Hy}$ pertension, 38, 1349-1354. doi:10.1161/hy1201.096575

[5] Cohn, J.N. (1999) Vascular wall function as a risk marker for cardiovascular disease. Journal of Hypertension, 17, S41-S44.

[6] Lehmann, E.D. (1999) Clinical value of aortic pulse-wave velocity measurement. Lancet, 354, 528-529. doi:10.1016/S0140-6736(99)00179-8

[7] Song, B.G., Park, J.B., Cho, S.J., et al. (2009) Pulse wave velocity is more closely associated with cardiovascular risk than augmentation index in the relatively low-risk population. Heart and Vessels, 24, 413-418. doi:10.1007/s00380-009-1146-1

[8] Yambe, T., Yoshizawa, M., Saijo, Y., et al. (2004) Brachioankle pulse wave velocity and cardio-ankle vascular index (CAVI). Biomedicine and Pharmacotherapy, 58, S95-S98.

[9] Shirai, K., Utino, J., Otsuka, K., et al. (2006) A novel blood pressure-independent arterial wall stiffness parameter: Cardio-Ankle vascular index (CAVI). Journal of
Atherosclerosis and Thrombosis, 13, 101-107. doi:10.5551/jat.13.101

[10] Lorenz, M.W., Markus, H.S., Bots, M.L., et al. (2007) Prediction of clinical cardiovascular events with carotid intima-media thickness: A systematic review and metaanalysis. Circulation, 115, 459-467. doi:10.1161/CIRCULATIONAHA.106.628875

[11] Yeboah, J., Folsom, A.R., Burke, G.L., et al. (2009) Predictive value of brachial flow-mediated dilation for incident cardiovascular events in a population-based study: The multiethnic study of atherosclerosis. Circulation, 120, 502-509. doi:10.1161/CIRCULATIONAHA.109.864801

[12] Yamasaki, Y., Kawamori, R., Matsushima, H., et al. (1994) Atherosclerosis in carotid artery of young IDDM patients monitored by ultrasound high-resolution B-mode imaging. Diabetes, 43, 634-639. doi:10.2337/diabetes.43.5.634

[13] Hoshida, S., Miki, T., Nakagawa, T., et al. (2011) Different effects of isoflavones on vascular function in premenopausal and postmenopausal smokers and nonsmokers: NYMPH study. Heart and Vessels, 26, 590-595. doi:10.1007/s00380-010-0103-3

[14] Morrison, K.M., Dyal, L., Conner, W., et al. (2010) Cardiovascular risk factors and non-invasive assessment of subclinical atherosclerosis in youth. Atherosclerosis, 208, 501-505. doi:10.1016/j.atherosclerosis.2009.07.034

[15] Hayaishi-Okano, R., Yamasaki, Y., Katakami, N., et al. (2002) Elevated C-reactive protein associates with earlystage carotid atherosclerosis in young subjects with type 1 diabetes. Diabetes Care, 25, 1432-1438. doi:10.2337/diacare.25.8.1432

[16] Fitch, K.V., Stavrou, E., Looby, S.E., et al. (2011) Associations of cardiovascular risk factors with two surrogate markers of subclinical atherosclerosis: Endothelial function and carotid intima media thickness. Atherosclerosis, 217, 437-440. doi:10.1016/j.atherosclerosis.2011.04.009

[17] Ross, R. (1986) The pathogenesis of atherosclerosis-An update. New England Journal of Medicine, 8, 488-500. doi:10.1056/NEJM198602203140806

[18] Celermajer, D.S., Sorensen, K.E., Gooch, V.M., et al. (1992) Non-Invasive detection of endothelial dysfunction in children and adults at risk of atherosclerosis. Lancet, 340, 1111-1115. doi:10.1016/0140-6736(92)93147-F

[19] Benjamin, E.J., Larson, M.G., Keyes, M.J., et al. (2004) Clinical correlates and heritability of flow-mediated dilation in the community. The Framingham Heart Study. Circulation, 109, 613-619. doi:10.1161/01.CIR.0000112565.60887.1E

[20] Fox, K., Borer, J.S., Camm, A.J., et al. (2007) Resting heart rate in cardiovascular disease. Journal of American College of Cardiology, 50, 823-830. doi:10.1016/j.jacc.2007.04.079

[21] Celermajer, D.S., Sorensen, K.E., Bull, C., et al. (1994) Endothelium-dependent dilation in the systemic arteries of asymptomatic subjects relates to coronary risk factors and their interaction. Journal of American College of Cardiology, 24, 1468-1474. doi:10.1016/0735-1097(94)90141-4

[22] Gokce, N., Holbrook, M., Hunter, L.M., et al. (2002) Acute effects of vasoactive drug treatment on brachial 
artery reactivity. Journal of American College of Cardiology, 40, 761-765. doi:10.1016/S0735-1097(02)02034-X

[23] Custodis, F., Baumhäkel, M., Schlimmer, N., et al. (2008) Heart rate reduction by ivabradine reduces oxidative stress, improves endothelial function, and prevents atherosclerosis in apolipoprotein E-deficient mice. Circulation, 117, 2377-2387.

doi:10.1161/CIRCULATIONAHA.107.746537

[24] Hutcheson, I.R. and Griffith, T.M. (1991) Release of endothelium-dereived relaxing factor is modulated both by frequency and amplitude of pulsatile flow. American Journal of Physiology, 261, 257-262.

[25] Harris, K.F. and Matthews, K.A. (2004) Interactions between autonomic nerve system activity and endothelial function: A model for the development of cardiovascular disease. Psychosomatic Medicine, 66, 153-164. doi:10.1097/01.psy.0000116719.95524.e2

[26] Celermajer, D.S., Sorensen, K.E., Spiegelhalter, D.J., et al. (1994) Aging is associated with endothelial dysfunction in healthy men years before the age-related decline in women. Journal of American College of Cardiology, 24, 471-476. doi:10.1016/0735-1097(94)90305-0

[27] Williams, M.R., Westerman, R.A., Kingwell, B.A., et al. (2001) Variations in endothelial function and arterial compliance during the menstrual cycle. Journal of Clinical Endocrinology and Metabolism, 86, 5389-5395. doi:10.1210/jc.86.11.5389 